\title{
Properties and Reinforcing Mechanism of Cellulose Reinforced Polyvinyl Alcohol Hydrogel Membranes
}

\author{
Huafeng Peng \\ College of Material Science and Engineering, \\ Donghua University, \\ Shanghai, China
}

\author{
Shaopeng Wang ${ }^{1}$ \\ College of Material Science and Engineering, \\ Donghua University, \\ Shanghai, China \\ China Textile Academy, Beijing, China \\ phfhaisha@163.com
}

\begin{abstract}
The novel cellulose reinforced Polyvinyl Alcohol (PVA) composite hydrogel membranes were prepared by means of immersion-precipitation phase transformation, taking the ionic liquid, 1-butyl-3-methylimidazolium acetate $\left([\mathrm{Amim}]^{+} \mathrm{Cl}^{-}\right)$ as solvent. The prepared hydrogels were characterized by Fourier transform infrared spectrum (FT-IR), X-ray diffraction measurements (XRD) and Scanning electron microscopy (SEM). The results revealed that a novel compact dual-network structure was formed between the cross-linked cellulose network and PVA, the formed of aggregate sheets by the hydrophobic action of cellulose will also be physical cross-linking network junctions besides the hydrogen bonds interactions in the hydrogel, and this is also one of the other reinforce factors to the PVA hydrogel. It is believed that this is an effective manner to reinforce PVA hydrogel materials.
\end{abstract}

Keywords—hydrogel; cellulose; polyvinyl Alcohol; reinforcing

\section{INTRODUCTION}

Poly vinyl alcohol (PVA) is a synthetic water-soluble polymer. Due to its good biocompatibility, stability, porous structure, low friction coefficient and the moisture content of similar to the body's tissues [1,2], the Polyvinyl alcohol hydrogel has been extensively investigated as promising applications in tissue replacement and repair materials, drug carrier, cartilage and skin substitutes, skin wound dressings and scaffold of cell culture, etc. Nonetheless, PVA hydrogels can hardly meet some high requirements of tissue replacement due to its unsatisfied mechanical properties $[3,4]$. Therefore, at present, the main research is to improve the mechanical properties of PVA hydrogels, meanwhile keep the original characters. Therefore, to improve the mechanical properties of the PVA hydrogel by incorporating reinforce material into the PVA hydrogel to form the composite hydrogel materials, meanwhile maintaining its biocompatibility has been received substantial attention in the past years [5-8] Reinforcing material of PVA hydrogel used as tissue requires good biocompatibility, besides having good mechanical properties.

Cellulose is the most abundant natural biologically renewable polymer on earth with numerous applications, has excellent mechanical property and some unique features such as hydrophily, optical activity, biocompatibility and biofunctionability, etc $[9,10]$. So that, cellulose is a kind of ideal reinforcing material for PVA hydrogel. PVA is a semicrystalline polymer bearing plenty of hydroxyl groups which produce inter- and intra-molecular hydrogen bonding. Since both the polymers are rather hydrophilic, strong molecular interactions (hydrogen bonding or van der Waals forces) between the cellulose and PVA can be expected.

The main objective of the present study is to prepare serial cellulose reinforced PVA hydrogels with various rations of cellulose based on the dissolution of cellulose and PVA in suitable ionic liquid, the morphological features, mechanical properties were to characterized, and the possible reinforced mechanism of the cellulose to the PVA hydrogel was discussed.

\section{EXPERIMENTAL SECTION}

Prior to the experiment, the cellulose and PVA were dried at $40^{\circ} \mathrm{C}$ in a vacuum oven for $5 \mathrm{hr}$. All dissolution experiments were performed in a three-necked flask by addition of the $8 \mathrm{wt} \%$ dried cellulose or PVA flakes in ionic liquid at room temperature and the temperature of the dissolving process was controlled by an oil bath at $70^{\circ} \mathrm{C}$ under vacuum conditions, with a mechanical stirrer, until transparent solution was prepared. The obtained cellulose and PVA solution with different weight ratios were blended and stirring continuously for 3 hours, five distinct cellulose/PVA solutions were prepared with different cellulose contents $0,10 \%, 30 \%$ and $50 \%$, denoted as a, b, c and d. The pure cellulose hydrogel (e) was prepared for comparisons.

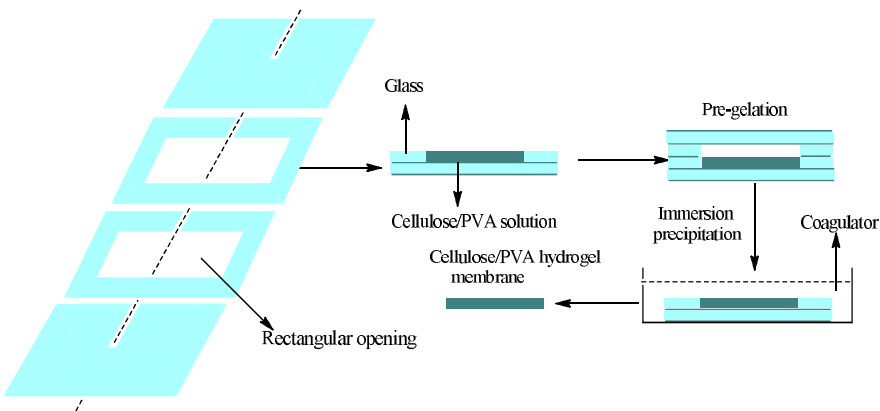

Fig. 1. Preparation procedures for cellulose reinforced PVA hydrogel membranes with ionic liquids.

The transparent solution was cast into the assembly molds which consist of four thin quartz glasses; the thickness of the middle piece is $1 \mathrm{~mm}$, with a rectangular opening $(100 \mathrm{~mm}$ $\mathrm{x} 100 \mathrm{~mm}$ ). A sealing container was formed to prevent water absorption during the pre-gelation. After it was kept under pre-gelation conditions $\left(-20^{\circ} \mathrm{C}, 12 \mathrm{hours}\right)$, the upper quartz 
glass was removed, both solution and the mold were immersed in water coagulant bath to give a cellulose hydrogel membrane. The preparation process is shown in Fig. 1. The cellulose hydrogel membranes were washed and soaked with water several times and saved in deionized water. In order to prevent the shrinkage of the hydrogel membranes during the drying process, a Freeze-drier was used to freeze-dry the hydrogel membranes after they were previously frozen using liquid nitrogen. A high vacuum $(0.010$ mbar) and a temperature of $-89^{\circ} \mathrm{C}$ were employed during the drying process. The samples were allowed to rest at these conditions for $72 \mathrm{~h}$. Finally the dried membranes were stored in desiccators to remove any residual traces of water. The morphological features and physical properties of the hydrogel membranes dried by lyophilization will be discussed.

\section{EXPERIMENTAL RESULTS AND DISCUSSION}

\section{A. FT-IR Analysis of Composite Membranes}

Reinforcing mechanism of the cellulose reinforced PVA hydrogel can be attributed to strong interactions via hydrogen bonds between the functional groups $(-\mathrm{OH})$ of the cellulose and PVA. FT-IR spectroscopy was used to examine these interactions between cellulose and PVA. The results of the FT-IR spectra of the cellulose reinforced PVA membranes are presented in Fig. 2. The large bands at 3300$3500 \mathrm{~cm}^{-1}$ (assigned to O-H stretching), near $2925 \mathrm{~cm}^{-1}$ (assigned to $\mathrm{C}-\mathrm{H}$ stretching) and about $1050 \mathrm{~cm}^{-1}$ (assigned to $\mathrm{C}-\mathrm{O}$ stretching) were found for all characterized materials. It suggests that any interactions between cellulose and PVA are physical rather than chemical in origin. It can be seen that the characteristic bands of cellulose and PVA are present in the spectra of their composites. The shifting of above mentioned bands indicate hydrogen bond interactions between the cellulose and PVA. The $1419 \mathrm{~cm}^{-1}$ band assigned to $-\mathrm{CH}_{2}$ stretching of cellulose crystalline II structure [11, 12], the stronger and shifting of this band indicate that the aggregation structure of cellulose crystalline II and the intraintermolecular hydrogen bonding involves the $\mathrm{O}$ of $\mathrm{C6}$ is destroyed.

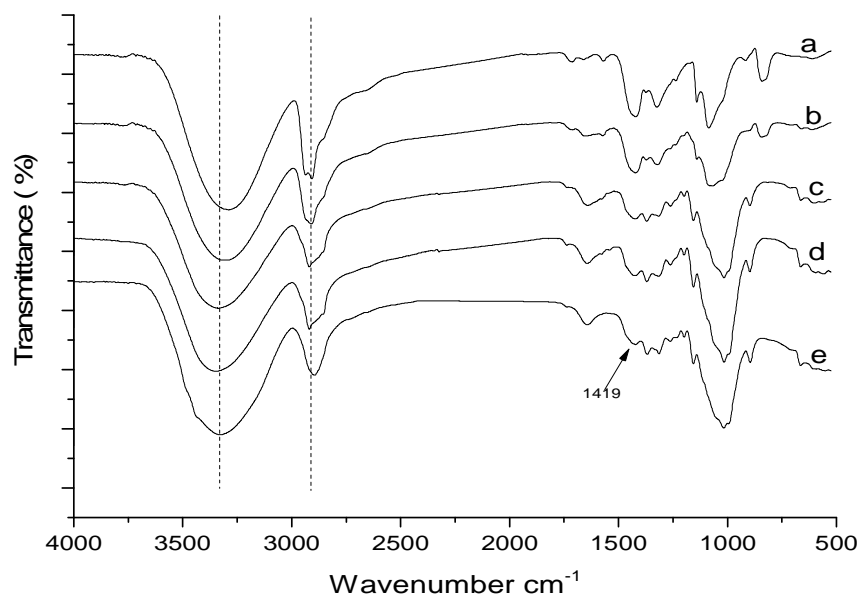

Fig. 2. FT-IR spectra for PVA (a), cellulose reinforced PVA b, c and d with cellulose content $10 \%, 30 \%$ and $50 \%$ and cellulose (e).

\section{B. Mechanical Properties Analysis}

Mechanical properties are important index to evaluate the application of the materials. The mechanical properties of the cellulose reinforced PVA hydrogel membranes with various cellulose contents were evaluated. Fig. 3 shows the tensile strength and elongation at break of cellulose reinforced PVA hydrogel membranes. The increasing of cellulose weight ratio is beneficial to improve the tensile strength and elongation at break of the composite hydrogel membranes. With $10 \mathrm{wt} \%$ cellulose, the tensile strength of composite hydrogels increased by more than $450 \%$ from $0.095 \mathrm{MPa}$ to $0.43 \mathrm{MPa}$. The enhancement of mechanical properties can be mainly attributed to the inherent excellent strength of cellulose and the interactions, specifically, hydrogen bonds formed between cellulose and PVA. At the high cellulose concentration $(50 \mathrm{wt} \%)$, the increment of elongation at break was not so obvious as compared with $30 \mathrm{wt} \%$, a possible reason is that the cellulose can simultaneously form hydrogen bonds with cellulose and PVA, which acted as physical crosslinking points in the composite hydrogels, at low cellulose concentration, the new added cellulose mainly formed the intermolecular interactions between the cellulose and PVA molecules and replaced the original interactions between the PVA molecules; when the cellulose concentration reached a certain ratio, the new added cellulose mainly formed the intermolecular interactions of cellulose molecules, replaced the original interactions between the cellulose and PVA molecules.

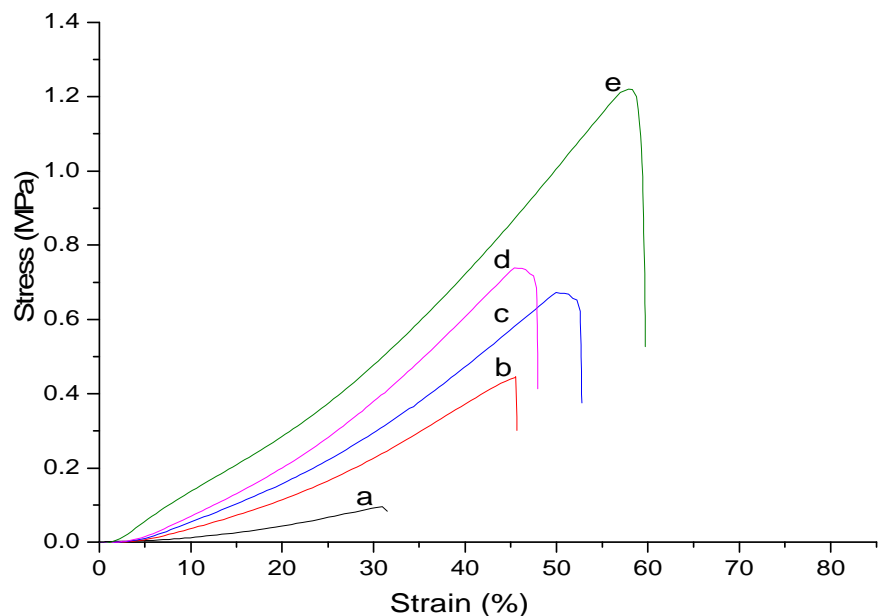

Fig. 3. Representative tensile stress-strain curves of the hydrogel membranes for PVA (a), cellulose reinforce PVA hydrogel b, c and d with cellulose content $10 \%, 30 \%, 50 \%$ and cellulose (e).

\section{X-ray Diffraction Analysis}

X-ray diffraction patterns of freeze-dried hydrogel membranes for the regenerated cellulose, PVA, cellulose reinforce PVA hydrogel are shown in Fig. 4. The diffraction pattern of the neat PVA exhibits a typical crystalline peak at $2 \theta=20.8^{\circ}$, corresponding to the $\left(\begin{array}{lll}1 & 0 & 1\end{array}\right)$ plane of semicrystalline PVA [13]. The XRD pattern of neat regenerated cellulose shows two typical diffraction peaks at $2 \theta=12.8^{\circ}$ and $20.8^{\circ}$. The peak at $2 \theta=12.8^{\circ}$ originates from the cellulose crystalline plane $\left(\begin{array}{lll}1 & 0 & 1\end{array}\right)$, and the peak at $2 \theta=23.1^{\circ}$ originates from the cellulose crystalline plane $(10 \overline{\mathbf{1}})$ and (002) [14-16], which indicated that the regenerated 
cellulose membrane is cellulose II. The characteristic diffraction peaks of PVA disappeared with the cellulose contents increasing, suggesting that the crystal structures of PVA were not maintained in the composites due to good miscibility of cellulose and PVA. Mean-while, a new diffraction peak arises and disappears at about $2 \theta=27^{\circ}$ with the increasing of cellulose contents, the diffractograms of the composites are obviously not the overlapping of the characteristic peaks of the two components, especially in the samples $\mathrm{c}$ and $\mathrm{d}$. This behavior may be explained by the presence of interactions between cellulose and PVA through hydrogen bonding might disrupt the ordered aggregates and crystalline structures of the polymers.

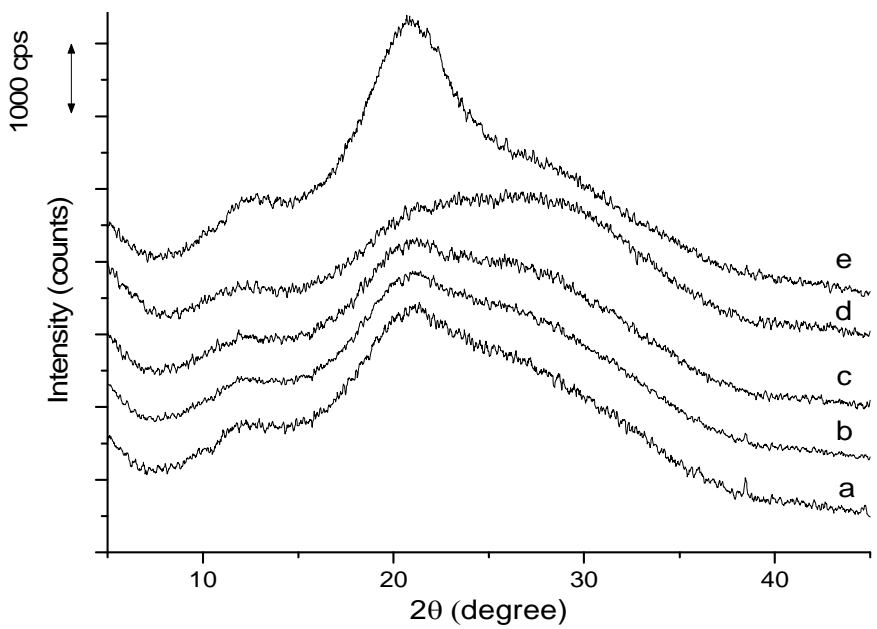

Fig. 4. WAXD comparison of PVA (a), cellulose reinforced PVA b, c and d with cellulose content $10 \%, 30 \%, 50 \%$ and cellulose (e).

\section{SEM Analysis}

The fracture surface SEM micrographs obtained from freeze dried cellulose, PVA and cellulose reinforced PVA hydrogel are presented in Fig. 5. The morphology of the cellulose reinforced is quite different from that of neat PVA and depends on the cellulose weight percentage. With the increase of cellulose content in the composites, the rob-like structures in surface were appeared gradually, the length of the rob-like structures ranging from $100 \mathrm{~nm}-300 \mathrm{~nm}$. This morphology transformation may be explained by considering as follows: unlike the PVA, cellulose is amphiphilic, i.e. contains both polar and nonpolar groups/segments/sides [1720]. The important evidence for amphiphilicity of cellulose is the helix structure, due to intra- and intermolecular hydrogen bonding, there is a formation of rather flat ribbons, with various sides that differ markedly in their polarity [21]. Therefore, due to the exclusion effect of the polar solvent, the hydrophobic sides would firs to stick to each other in order to reduce the non-polar surface area by adjusting the self-geometrical structure in the immersion-precipitation phase transformation and freeze-dried process, the rob-like structure are the aggregates of this kind of piles. Then these aggregates contact and adhered to each other by hydrogen interactions to form the gel membrane.
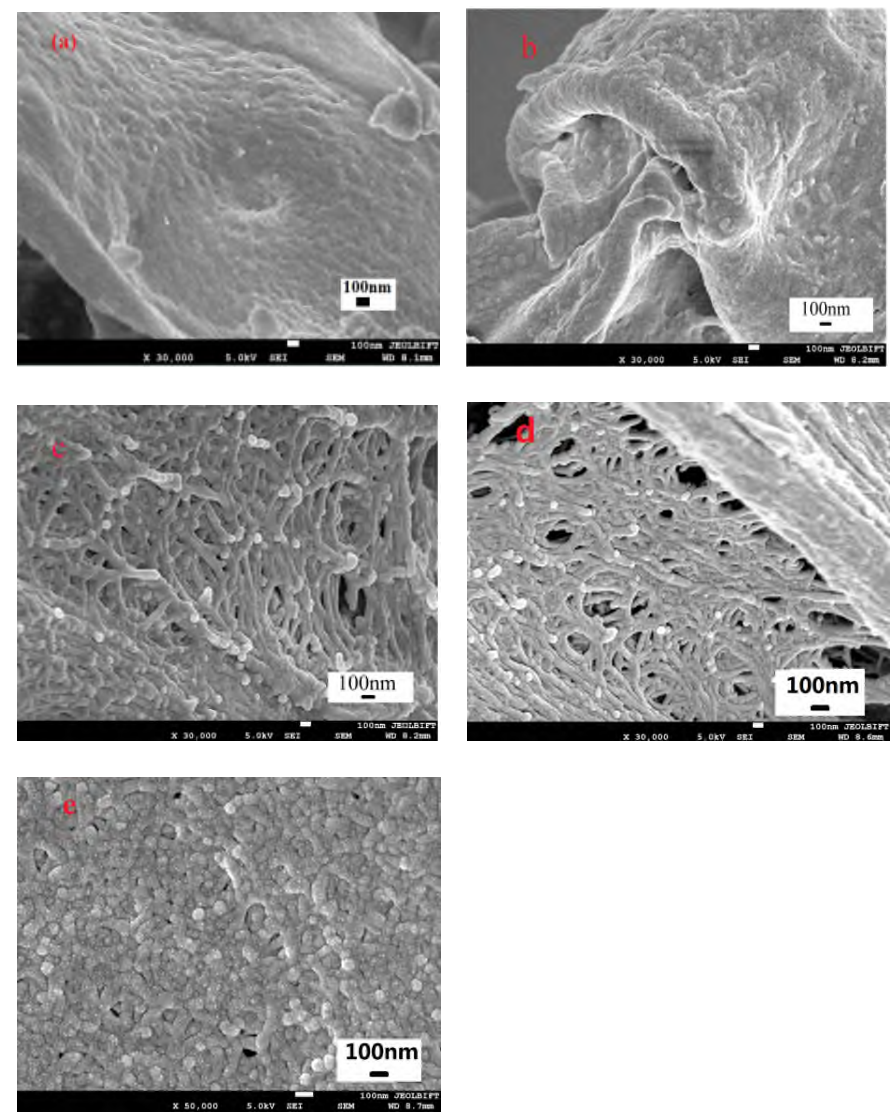

Fig. 5. SEM micrographs of cross-section for freeze-dried PVA and cellulose reinforced hydrogel a, b, c and d with cellulose content $10 \%, 30 \%, 50 \%$ and cellulose hydrogel e

\section{E. Reinforcing Mechanisms of Cellulose for PVA Hydrogel}

The above results suggested that the reinforcing mechanisms of cellulose in the PVA hydrogel are mainly involved two aspects, first, a large number of hydroxyl groups in the molecular chains of both the cellulose and PVA enhanced molecular interactions between the cellulose and PVA because of forming the hydrogel junctions that are mainly produced by interaction of hydrogen bonding; Cellulose is amphiphilic, due to the exclusion effect of the polar solvent (water), the hydrophobic sides would stick to each other to form aggregate sheets in order to reduce the non-polar surface area by adjusting the self-geometrical structure in the immersion-precipitation phase transformation process. The formed aggregate sheet should be micelle structure, in which, inward is the hydrophobic structure and outward is hydrophilic structure, thereby the surface energy of the micelle is reduced in the solution, the hydrophilic PVA molecules are more easily coated on the outside of the formed micelles. The formed aggregate sheets could also be physical cross-linking network junctions beside the hydrogen bonds interactions in the pregelation.

Through above characterizations and analysis, the reinforcing of cellulose can be considered that, at the low cellulose contents, the reinforcing mechanism is mainly because excellent mechanical property of cellulose and the hydrogen bond between the cellulose and the PVA molecules; 
at the high cellulose contents, the reinforcing mechanism also involved the rod-like aggregate sheet which formed by the hydrophobic action of cellulose, all the hydrogen bonds and aggregates act as the junctions in the formed cellulose reinforced PVA hydrogels, and the mechanism as below (Fig. $6)$.

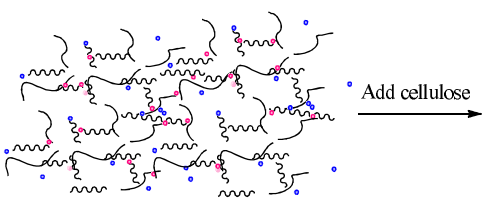

Low cellulose content

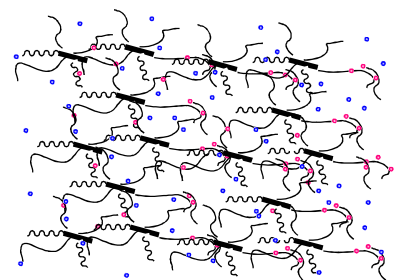

High cellulose content
- Aggregate sheet $ح$ Cellulose num PVA ${ }^{\circ}$ Hydrogen bond ${ }^{\circ}$ Water

Fig. 6. Possible mechanism of cellulose reinforced PVA hydrogel.

\section{CONCLUSIONS}

In this paper, the cellulose reinforced PVA hydrogels were prepared taking ionic liquid $[\mathrm{Amim}]^{+} \mathrm{Cl}^{-}$as solvent. It shows relatively good miscibility and strong molecular interaction between cellulose and PVA is due to the presence of large amounts of hydroxyl groups in the molecular chains of cellulose and PVA, in which hydrogel junctions are produced under the interaction of hydrogen bonds. The presence of rod-like structures can be used as evidence of aggregates formed by the hydrophobic action of cellulose, indicating that the formed of aggregate sheets will also be physical cross-linking network junctions besides the hydrogen bonds interactions in the hydrogel. Compared with the neat PVA, the mechanical properties of the cellulose reinforced PVA hydrogel membrane show a significant improvement, depending on the ratio of cellulose in the PVA hydrogel. The mechanical properties of cellulose reinforced PVA hydrogels are enhanced by the hydrogel junctions via the hydrogen bonds and the network structure of the aggregate sheets of cellulose. It is believed that this is an effective manner to prepare the high strength PVA hydrogel materials.

\section{ACKNOWLEDGEMENTS}

The project was supported by grants from the Natural Science Foundation of China (No. 21471030)

\section{REFERENCE}

[1] M. Kobaysshi, Y.S. Chang and M. Oka, "A two year in vivo study of polyvinyl alcohol-hydrogel (PVA-H) artificial meniscus". Biomater. vol. 26, pp. 3243-3248, 2005.

[2] M.R. Hwang, J.O. Kim, J.H. Lee, Y. Kim, J.H. Kim, S.W. Chang, S.J. Jin, J.A. Kim, W.S. Lyoo, S.S. Han, S.K. Ku, C.S. Yong and H.G. Choi, "Gentamicin-loaded wound dressing with polyvinyl alcohol/dextran hydrogel: gel characterization and in vivo healing evaluation," AAPS Pharm. Sci. Tech. vol. 11, pp. 1092-1103, 2010.

[3] K. Ushio, M. Oka, S. Hyon, T. Hayami, S. Yura and K. Matsumura, "Attachment of artificial cartilage to underlying bone.” J. Biomed. Mater Res, Part B: vol. 68B, pp. 59-68, 2004.

[4] M.R. Hwang, J.O. Kim, J.H. Lee, Y. Kim, J.H. Kim, S.W. Chang, S.J. Jin, J.A. Kim, W.S. Lyoo, S.S. Han, S.K. Ku, C.S. Yong and H.G. Choi, "Gentamicin-loaded wound dressing with polyvinyl alcohol/dextran hydrogel: gel characterization and in vivo healing evaluation." AAPS Pharm. Sci. Tech. vol. 11, pp. 1092-1103, 2010.
[5] J.S. Bach, F. Detrez, M. Cherkaoui, S. Cantournet, D.N. Ku and L. Corté, "Hydrogel fibers for ACL prosthesis: design and mechanical evaluation of PVA and PVA/UHMWPE fiber constructs." J. Biomech. vol. 46, pp. 1463-1470, 2013

[6] Y.X. Ma, Y.D. Zheng, X.S. Huang, T.F. Xi, X.D. Lin, D.F. Han and W.H. Song, "Mineralization behaviorand interface properties of BGPVA/bone comsite inplantsin simulated boday fluid Biomed." Mater. vol. 5, pp. 25003-1-025003-8, 2010.

[7] Y.D. Zheng, H.X. Lv, Y.J. Wang, H.D. Lu, L. Qing and T.F. Xi, "Performacne of novel bioactive hybrid hydrogels in vitro and in vivo used for artificial cartilage." Biomed. Mater. vol. 4, pp. 15015-1015015-7, 2009.

[8] Y.Y. Huang, Y.D. Zheng, W.H. Song, Y.X. Ma, J. Wu and L.Z. Fan, "Poly (vinyl pyrrolidone) wrapped multi-walled carbon nanotube/poly (vinyl alcohol) composite hydrogels." Composites, vol. 42, pp. 13981405, 2011.

[9] A.L. ABrown, "On an acetic ferment which forms cellulose." Journal of the Chemical Society. J. Chem. Soc. vol. 49, pp. 432-439, 1886.

[10] D. Klemm, B. Heublein and H.P. Fink, "Cellulose: fascinating biopolymer and sustainable raw material." Angew. Chem. Int. Ed. vol. 44, pp. 3358-3393, 2005.

[11] S.Y. Oh, D. Yoo, Y. Shin, H.C. Kim, H.Y. Kim, Y.S. Chung, W.H. Park and J.H. Youk, "Crystalline structure analysis of cellulose treated with sodium hydroxide and carbon dioxide by means of X-ray diffraction and FTIR spectroscopy," Carbohydr. Polym. vol. 340, pp. 2376-2391, 2005.

[12] F. Carrillo, X. Colom and J.J. Sunol, "Structural FTIR analysis and thermal characterisation of lyocell and viscose-type fibres." Eu. Polm. J. vol. 40, pp. 2229-2234, 2004.

[13] X.W. Sun, C.H. Lu, Y. Liu, W. Zhang and X.X. Zhang, "Melt-processed poly (vinyl alcohol) composites filled with microcrystalline cellulose from waste cotton fabrics." Carbohydr. Polym. vol. 101, pp. 642-649, 2014.

[14] H.S. Qi, J. Cai, L. Zhang and S. Kuga, "Properties of films composed of cellulose nanowhiskers and a cellulose matrix regenerated from alkali / urea solution." Biomacromolcules, vol. 10, pp. 1597-1602, 2009.

[15] B.J.C. Duchemin, A.P. Mathew and K. Oksman, "All-cellulose composites by partial dissolution in the ionic liquid 1-butyl-3methylimidazolium chloride." Composites, vol. 40, pp. 2031-2037, 2009

[16] N. Hameed and Q. Guo, "Blend films of natural wool and cellulose prepared from an ionic liquid." Cellulose, vol. 17, pp. 803-813, 2010.

[17] L. Bjom, K. Gunnar and S. Lars, "On the mechanism of dissolution of cellulose,” J. Mol. Liq. vol. 156, pp. 76-81, 2010.

[18] H. Miyamoto, M. Umemura, C. Yamane, K. Ueda and K. Takahashi, "Structural reorganization of molecular sheets derived from cellulose II by molecular dynamics simulations." Carbohyd. Res. vol. 344, pp. 1085 1094, 2011.

[19] H. Miyamoto, C. Yamane, M. Seguchi, K. Ueda and K. Okajima, "Supermolecular structure of cellulose/amylose blends prepared from aqueous $\mathrm{NaOH}$ solutions and effects of amylose on structural formation of cellulose from its solution." Carbohyd. Res. vol. 346, pp. 807-814, 2011.

[20] C. Yamane, R. Hirase, H. Miyamoto, S. Kuwamoto and Y. Yuguchi, "Mechanism of structure formation and dissolution of regenerated cellulose from cellulose/aqueous sodium hydroxide solution and formation of molecular sheets deduced from the mechanism." Cellulose, vol. 22, pp. 2971-2982, 2015.

[21] K.U. Goss and R.P. Schwarzenbach, "Rules of thumb for assessing equilibrium partitioning of organic compounds: Successes and pitfalls." J. Chem. Educ. vol. 80, pp. 450-455, 2003 